\title{
Out-of-plane artifact removal in photoacoustic imaging using transducer array displacement
}

H. N. Y. Nguyen, W. Steenbergen

H. N. Y. Nguyen, W. Steenbergen, "Out-of-plane artifact removal in photoacoustic imaging using transducer array displacement," Proc. SPIE 11077, Opto-Acoustic Methods and Applications in Biophotonics IV, 110770P (19 July 2019); doi: 10.1117/12.2526617

SPIE. Event: European Conferences on Biomedical Optics, 2019, Munich, Germany 


\title{
Out-of-plane artifact removal in photoacoustic imaging using transducer array displacement
}

\author{
H. N. Y. Nguyen*, W. Steenbergen \\ Biomedical Photonic Imaging Group, Faculty of Science and Technology, University of Twente, \\ P.O. Box 217, 7500 AE, Enschede, The Netherlands
}

\begin{abstract}
Current research has been extensively focusing on translating photoacoustic imaging into clinics using ultrasound handheld probes. However, a major drawback of these probes is the occurrence of artifacts which might lead to critical image misinterpretation. In-plane and out-of-plane artifacts are the two types of the artifacts in photoacoustic imaging. Recently we have reported a method for identifying reflection artifacts (in-plane artifacts). In this work, we propose a new method for removing out-of-plane artifacts by displacing the transducer array. Using transducer array displacement, out-of-plane artifacts can be de-correlated with in-plane image features and thus removed. We experimentally demonstrated this method with experiments in phantoms as well as in vivo. Results show that this is a promising approach for correcting out-of-plane artifacts.
\end{abstract}

\section{INTRODUCTION}

Over the past few years, numerous applications of photoacoustic imaging have been made showing potential of this technique in clinics [1-3]. One of approaches for applying PAI to clinics is integrating light source into an ultrasound (US) handheld probe. However, due to the limited view angle of the transducer array, artifacts might be present in acquired images. Artifacts in PAI might significantly reduce the quality of the images and lead to image misinterpretation [4-6]. Therefore, correcting artifacts in PAI is of importance.

There are two types of artifacts in PAI which are in-plane artifact (IPA) and out-of-plane artifact (OPA). Previously, we have reported a method for identifying and removing IPAs (reflection artifacts) using multi-wavelength excitation [7]. However, this method does not work for OPAs. In this work, we propose a new approach for removing OPAs using transducer array displacement. By axially displacing the transducer array, OPAs can be distinguished from in-plane image features and thus removed. Particularly, along the displacement, OPAs also axially move while in-plane image features remain at the same position. By comparing images pre- and post-displacement and keeping mutual features, OPAs can be removed. We experimentally evaluate the method with experiments in phantoms as well as in vivo. The results show that this is a potential method for correcting OPAs.

\section{MATERIALS AND METHOD}

The occurrence of OPAs in PAI happens when light beam excites a large volume in which some out of imaging plane absorbers are located. These absorbers are then excited and generate ultrasound waves. As a result, they might appear in acquired PA images as OPAs. They appear at depths equal to their distance to the transducer array. The distance obeys the Pythagorean formula for axial and elevation distances. However, in-plane absorbers appear at depths equal to their axial distance to the transducer array. As a consequence, if the transducer is axially displaced, in-plane features and OPAs can be differentiated.

A phantom made of 2 black absorbers embedded in agarose in a petri dish was used to mimic one in-plane absorber and one out-of-plane absorber. A solution of $2 \%$ Intralipid $20 \%$ in demi-water was used as a coupling medium. The probe was placed vertically above one absorber while the other absorber was positioned a few millimeters elevationally away from the probe.

*h.n.nguyen@utwente.nl,; phone +31 534891224

Opto-Acoustic Methods and Applications in Biophotonics IV, edited by Vasilis Ntziachristos,

Roger Zemp, Proc. of SPIE-OSA Vol. 11077, 110770P · (c) 2019 SPIE-OSA

CCC code: $1605-7422 / 19 / \$ 21 \cdot$ doi: $10.1117 / 12.2526617$

Proc. of SPIE-OSA Vol. 11077 110770P-1 
An experiment on a volunteer's finger was also carried out. A black ink mark was marked on the finger as an out-ofplane absorber mimicking a human spot or birthmark. The finger was placed in a water tank with demi-water used as a coupling medium.

A commercial US scanner MyLabOne (Esaote Europe BV, The Netherlands) reported in [7] was used for acquiring data. A US handheld probe which is similar to the one reported in [7] was used to detect US signals. The handheld probe was attached on a motorized translation stage (MTS50A-Z8, Thorlabs, Germany) which could translate along axial axis. Laser light was from an Opolette 532 (Laser2000, The Netherlands) at a wavelength of $790 \mathrm{~nm}$ and a repetition rate of $20 \mathrm{~Hz}$. The light was then coupled into a custom-made multi-mode optical fiber bundle (LightGuideOptics Germany $\mathrm{GmbH}$, Germany) with a core diameter of $6.5 \mathrm{~mm}$. The energy of the light at the output of the fiber bundle was $\sim 4.5$ $\mathrm{mJ} /$ pulse. The system was synched using a function generator (AFG 3102, Tektronix, Germany).

In our experiments, a distance of $5 \mathrm{~mm}$ of the probe displacement was performed while the fiber bundle was fixated. At each $1 \mathrm{~mm}$ of the displacement, 1 PA was acquired giving 6 PA images in total.

\section{RESULTS}

\section{Phantom}

Figure 1 shows acquired PA images along the probe displacement, $\Delta z$. In-plane and out-of-plane absorbers are clearly visible (features on the right and left hand side respectively). During the displacement, the OPA noticeably axially moves up while the in-plane feature basically remains at the same position. Some slight moves of the in-plane feature might be due to error when translating the probe or the probe not being perfectly axially displaced.
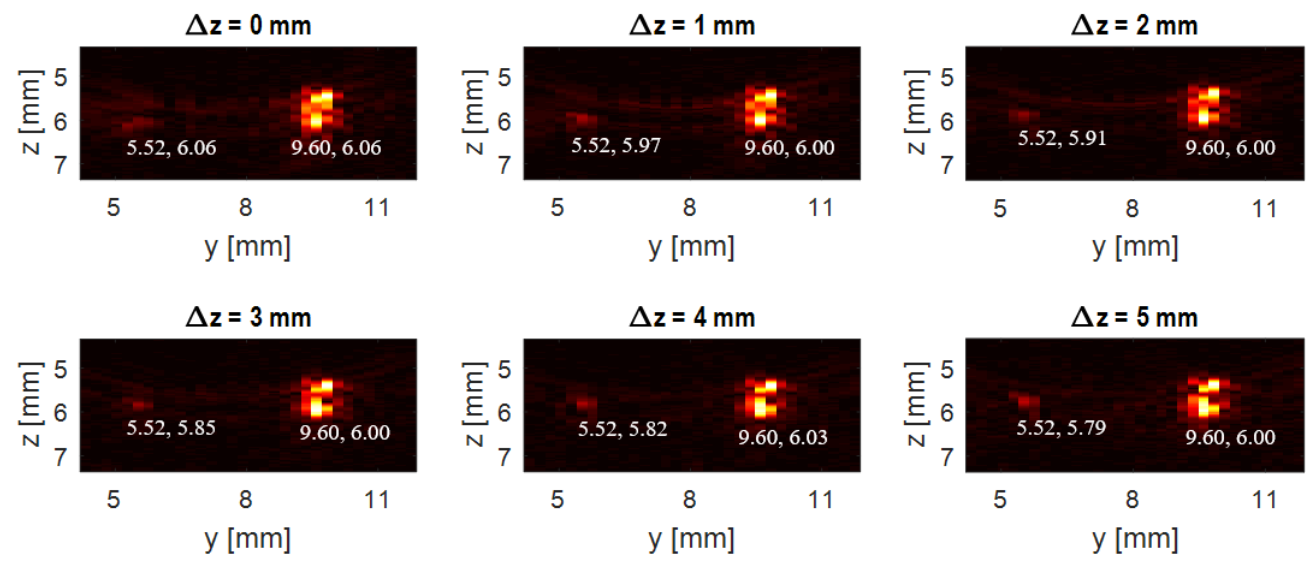

Figure 1. Acquired PA images along the probe displacement.

The OPA therefore can be decorrelated from the in-plane feature and removed. Figure 2 shows the result of this phantom experiment in which the OPA is completely removed.
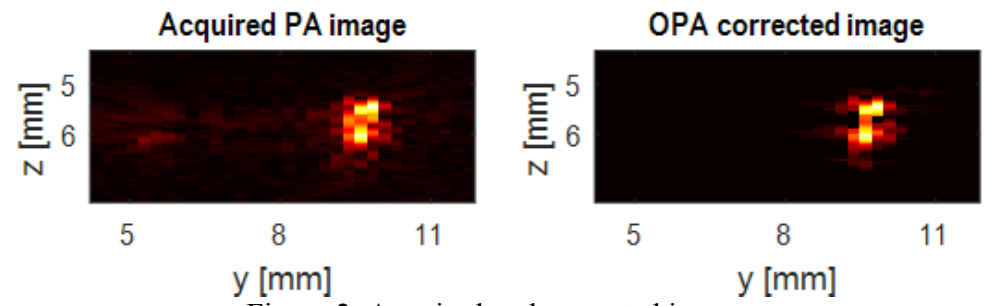

Figure 2. Acquired and corrected image.

\section{In vivo}

Figure 3 presents the result of an in vivo experiment showing a cross view of the volunteer's finger. OPAs of the ink mark is clearly present which looks like blood vessels (dashed blue circle). In the OPA corrected image, these OPAs are completely removed. it is worth noting that a curve at the skin is a reconstruction artifact. This type of reconstruction 
artifact is dependent on the distance to the transducer array. This is the reason why it is also reduced in the corrected image.
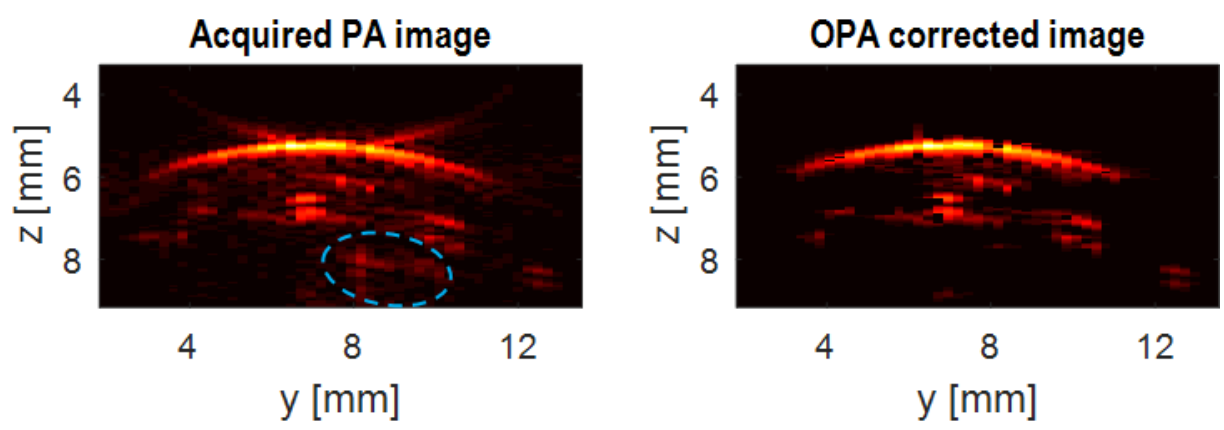

Figure 3. Acquired and corrected image.

\section{DISCUSSION AND CONCLUSION}

The proposed method can differentiate out-of-plane artifacts in PAI and thus correct them. We have experimentally demonstrated this method for a proof of concept. In addition, we have also shown that this method can reduce reconstruction artifacts. However, this method does not work for in-plane artifacts. Combining this method with the previous method for in-plane artifacts will be our future work.

In our experiments, the fiber bundle was not displaced with the handheld probe. This was to assure that acquired images showed the same structure. In a configuration that the light source is also displaced, laser beam might not excite in-plane absorbers but some other out-of-plane structures. As a consequence, real in-plane image features might be removed.

The proposed method the transducer array must be axially displaced. In a clinical scenario, this requires significant experience or training of the users. However, a holder can be considered in this case.

\section{ACKNOWLEDGEMENTS}

The authors are grateful to Johan van Hespen and Tom Knop, University of Twente, for their help with experiment setup.

This work is financially supported by European Union's Horizon 2020 research and innovation programme under grant number H2020-731771.

\section{REFERENCES}

[1] K. S. Valluru, K. E. Wilson, and J. K. Willmann, "Photoacoustic Imaging in oncology: translational preclinical and early clinical experience," Radiology 280(2), 332-349 (2016).

[2] M. Heijblom, D. Piras, F. M. van den Engh, M. van der Schaaf, J. M. Klaase, W. Steenbergen, and S. Manohar, "The state of the art in breast imaging using the Twente Photoacoustic Mammoscope: results from 31 measurements on malignancies," Eur. Radiol. 26(11), 3874-3887 (2016).

[3] M. Heijblom, W. Steenbergen, and S. Manohar, "Clinical photoacoustic breast imaging: the twente experience," IEEE Pulse 6(3), 42-46 (2015).

[4] S. Preisser, G. Held, H. G. Akarçay, M. Jaeger, and M. Frenz, "Study of clutter origin in in-vivo epi-optoacoustic imaging of human forearms," J. Opt. 18(9), 094003 (2016).

[5] M. Jaeger, L. Siegenthaler, M. Kitz, and M. Frenz, "Reduction of background in optoacoustic image sequences obtained under tissue deformation," J. Biomed. Opt. 14(5), 054011 (2009).

[6] M. Jaeger, J. C. Bamber, and M. Frenz, "Clutter elimination for deep clinical optoacoustic imaging using localised vibration tagging (LOVIT)," Photoacoustics 1(2), 19-29 (2013).

[7] H. N. Y. Nguyen, A. Hussain, and W. Steenbergen, "Reflection artifact identification in photoacoustic imaging using multi-wavelength excitation," Biomed. Opt. Express 9(10), 4613-4630 (2018). 